\title{
A Smart Proxy for a Next Generation Web Services Transaction
}

\author{
Sunam Pradhan ${ }^{1,2}$ \\ ${ }^{1}$ School of Information Technology and \\ Mathematical Sciences, University of Ballarat \\ Mount Helen, Ballarat, Victoria, Australia \\ s.pradhan@ballarat.edu.au
}

\author{
Arkady Zaslavsky ${ }^{2}$ \\ ${ }^{2}$ Faculty of Information Technology, Monash \\ University \\ Caulfield, Victoria, Australia \\ Arkady.Zaslavsky@csse.monash.edu.au
}

\begin{abstract}
In this paper, we propose and describe sProxy - smart proxy, a software tool in Web Services transaction. sProxy acts as a gateway between transaction management systems and Web Services which implements a key abstraction of proxy management systems. This enables to perform transactions in the loosely coupled environment i.e. loose coupling among services. Proxies are useful to invoke Web Services to allow an easy programming model that facilitates the serialization and transmission of service invocations. Our proposed model supports relaxation of traditional ACID properties with existing commit and recovery protocols. The model works on non-ACID type of transactions which encapsulates Web Services. It also uses multithreading proxies to check and update transaction simultaneously. The proposed model solves the current problems with distributed computational activities which involves both transactions and Web Services. The proposed model is more abstract and generic as demonstrated in the paper.
\end{abstract}

\section{Introduction}

Web Services are an emerging technology for next generation Internet, which is a solution for current business needs. Basically Web Services provide distributed M2M services to client applications. It is expanding rapidly and all major software vendors are marketing their Web Services tools and applications.

Transaction is a core and vital element of any business. A transaction is a request from client application to a server which consists of two or more operations which has one logical function; this must be carried out in entirety or not at all. There are many challenges in Web Services transaction management namely, ACID properties are too restrictive, no proper concurrency control mechanism in distributed and heterogeneous level, poor security mechanism in Web Services and integrating existing components are difficult is huge scalability and interoperability challenges.

The purpose of this paper to describe how smart proxy can be used to perform transactions in loosely coupled environment such as Web Services. The paper proposes a new proxy based transactional model where proxy management system plays central role to manage and multithread transactions. It is very useful abstraction and it is practical.

In this paper, we present a three tier application, namely client application with transaction processing system as the first tier, sProxy as a middleware and Web Services, the third tier. Request is sent from a client application to the transaction processing system. With the help of the sProxy, query is processed and gets result from web servers. The approach has its own challenges and limitations, such as concurrency control, atomic commit protocol support, context-aware query, adaptability, scalability, interoperability etc. In this paper we are focused on commit protocols.

Major advantages of our approach are:
a. Loose coupling among services
b. Long running, interactive transactions
c. QoS can be defined by the user
d. Low cost model since sProxy acts as middleware and gateway between services and components.

\section{2. sProxy Transactional Model}

The model implements a proxy management system which has the capacity of managing and processing transactions hence named sProxy - smart proxy. It acts as a gateway between transaction management and Web Services. Our model is derived from proxy server model.

\section{sProxy Transaction Model}

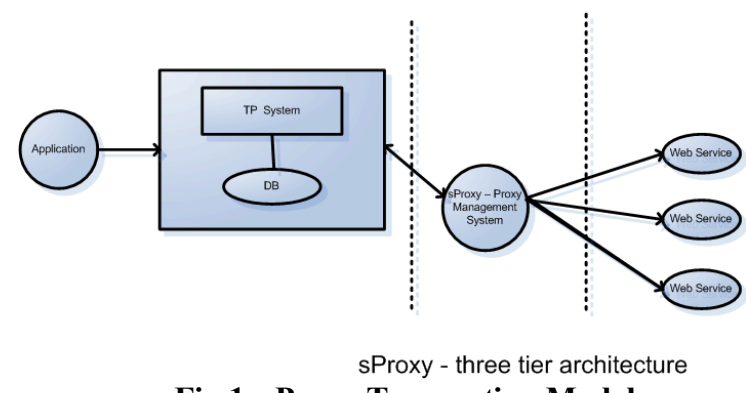

Fig 1. sProxy Transaction Model 
This model has programming, wrapping and composing features.

\subsection{Architectural Perspective}

The main idea is to introduce a proxy component as an intermediate layer between the transaction management and the Web services (see Fig 1). At runtime, proxy acts as wrapper, which means it wraps all the information required into packets and sends it to the Web Servers. Then proxy retrieves the result from the server and sends to Transaction Manager. Here all the activities done at Web services are hidden by the proxy. Interaction between transaction manager and the Web services are handled by the proxy which means the proxy is responsible for all communications which is handled by SOAP protocol.

Next step involves implementation of IDL (Interface Definition Language). This step is important in order to interact between transaction manager and the proxy. WSDL (Web Services Description Language) can be used as IDL. UDDI (Universal Discovery, Description and Integration) provides service directory for application to find the services.

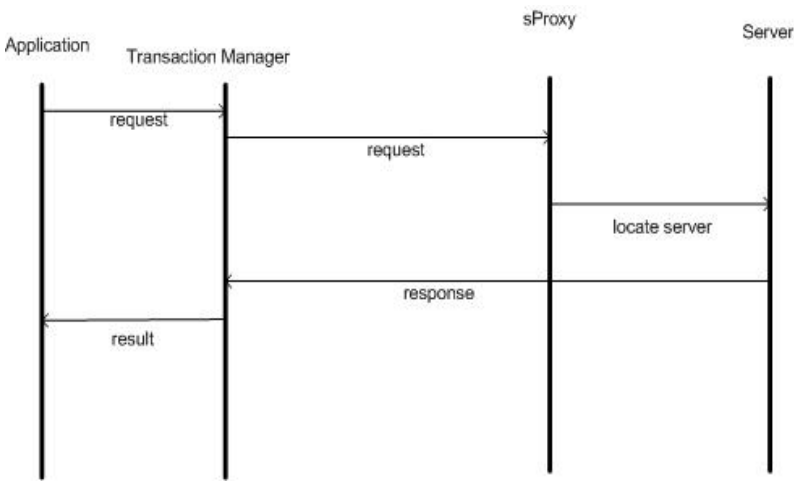

Fig 2. Sequence diagram for sProxy

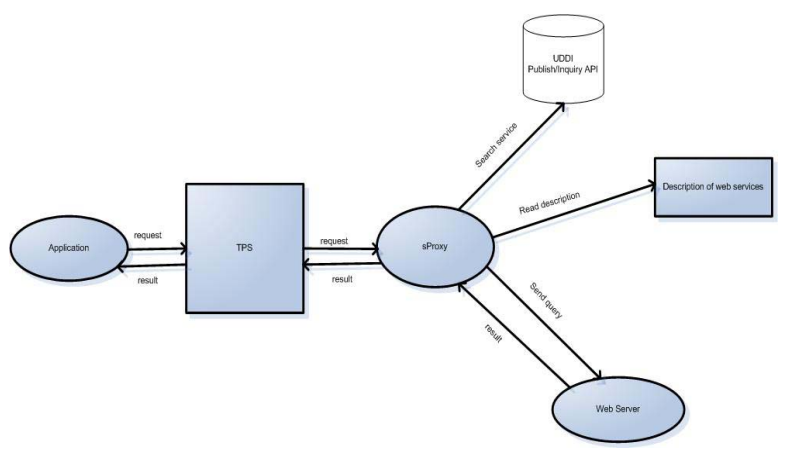

Fig 3. sProxy with UDDI

\subsection{Proxy Management System}

sProxy has traditional proxy properties, namely service, permissions, caching, routing, publishing, protocols, transparency, proxy chaining and logging [12].

As described in [7], the sProxy will have new edge services, programmability, configurability, adaptability, centralized or distributed system architecture, lifecycle support of applications, personalization, services activation and maintainability of software. In services, sProxy also determines stateful or stateless transactions. It deals with both web protocols and transactional protocols.

\subsection{Atomic transaction}

Atomic transaction is a transaction which is either committed or aborted i.e. all or nothing. Atomic transaction has ACID properties - Atomic, Consistent, Isolation and Durable. It is a technique for guaranteeing consistency in the presence of failures. When transaction is successful it is called committed and moved in a new state. When transaction is unsuccessful then it is rolled back, this is known as aborted. A coordinator is responsible for the outcome of a transaction that is either committed or rolled back. It communicates with participants involved in the transaction and work within the scope of the transaction. A transaction manager is responsible for managing multiple coordinators. Atomic transaction uses two-phase commit protocol for a transaction to make atomic. To avoid the blocking nature, distributed transaction uses three-phase commit. However, this is not adequate for long running transactions, where relaxation of the properties is required.

\subsubsection{Commit Protocol}

Commit Protocol ensures that the transaction is atomic. There are several commit protocols proposed in this area. Two phase commit protocol is also known as blocking protocol since resources are locked during the transaction so it is not suitable for distributed environment. Three phase commit protocol is non blocking protocol which suits for distributed environment.

\subsubsection{Transactional proxies}

The coordinator coordinates all resources involved in transaction and uses software transactional memory. For each transaction, sProxy creates transactional proxies and will be registered for the lifecycle of the transactions. This guarantees the ACID properties. It means proxy plays the implementation specific work to make transaction atomic [6].

\subsection{Programming}

It uses dynamic proxy classes to create a proxy class object for each transaction. Each SOAP method exposed by the service will be represented by a method in the 
proxy class. To access the Web service, instance of the proxy class is created and appropriate method is called.

\subsection{Wrapping}

A wrapper is a unique technique where new interface is created between existing technology/software and external environment. This replaces the original behaviour with extended behaviours. Wrapping is done with interposition, which involves creation of process between existing object. It introduces robustness in wrapped applications which includes fault tolerance, caching, security and authentication, firewall protection and Quality-of-service. Wrapped Web servers [13] have potential growth into groupware applications and environments, and dynamic information exchange.

\section{Implementation}

To further illustrate our concept of transaction on Web Services, We have considered Wine Planet scenario. To validate the proposed model, we will be using logging mechanism to log at runtime without modifying the application binary where logging behaviour can be controlled and edited by using a configuration file. Test cases are monitored. The prototype is in initial phase, where full features are yet to be implemented. We developed the prototype on the Windows operating system using Java with Apache Tomcat, MySql and Java Transaction server.

\subsection{Scenario - Wine Planet}

Consider a scenario where people buying wine from an ecommerce site. It consists of a huge content richness over a database of thousands of products or so and its related information. Information is very important because people really need information to make a purchasing decision. Two main tasks involved in this context are:

a. Customer ordering wine

b. Customer paying via Credit Card

\subsubsection{Business Transaction Scenarios}

We have used use case diagrams to understand and explore business transactions scenarios.

Client initiates transaction from the web browser as in Fig 8. When the client clicks on the link Wine list, information is requested from Java transaction server where it displays the list of wines (Fig 9). Client is able to get more information of wine by clicking on the icon info. Lets assume that that information is stored in different Web Services. In this case, information on Semillion wine is stored on the "ballarat.edu.au". sProxy sends the request to the specific web services and retrieves required information (Fig 10).

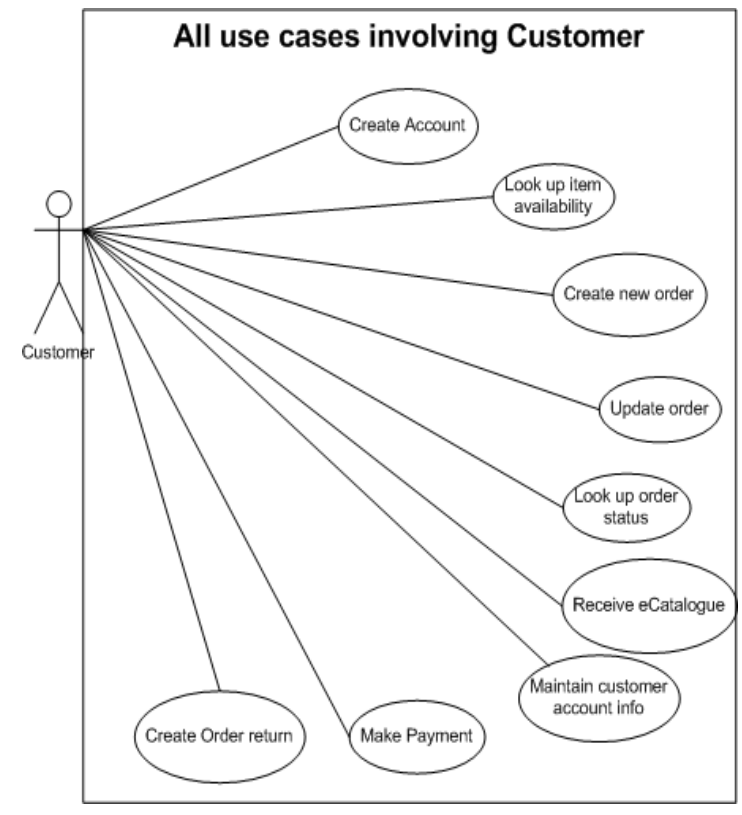

Fig 4. Customer use case

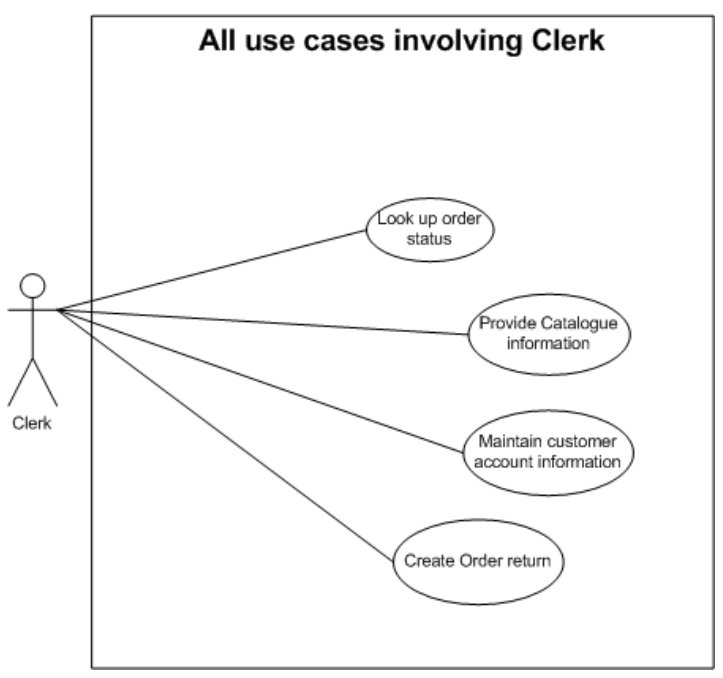

Fig 5. Clerk use cases

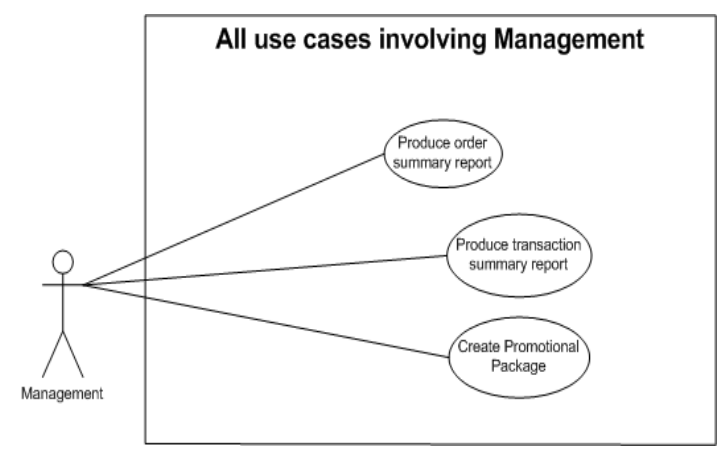

Fig 6. Management use cases 


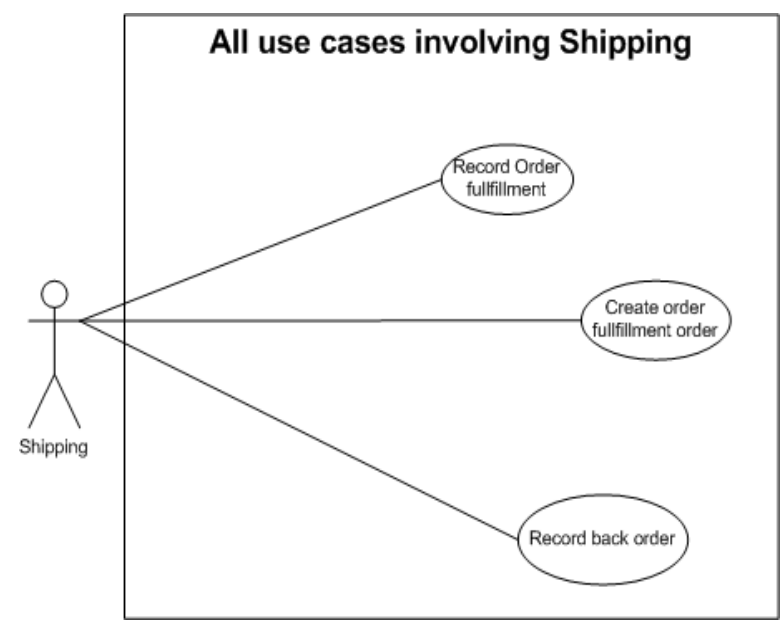

Fig 7. Shipping use cases

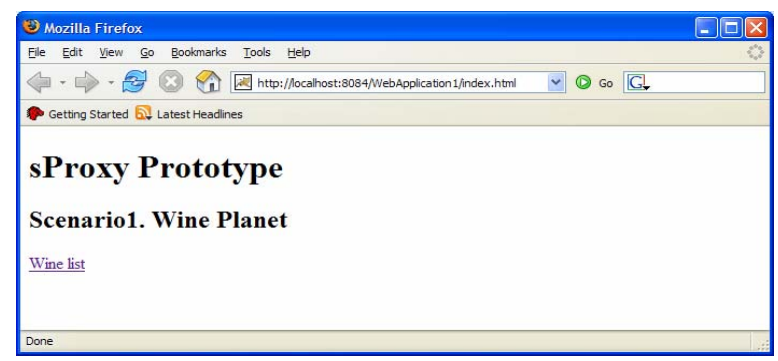

Fig 8. Client browser

\begin{tabular}{|c|c|c|c|c|}
\hline (3) sProxy Prototy & ype - Wine List - $M$ & Mozilla Firefox & & \\
\hline Ele Edit View $\mathrm{C}$ & Go Bookmarks Io & Lools Help & & \\
\hline$\Delta \cdot \Delta \cdot \sqrt{2}$ & y $x$ i & http://ocalhost:8084/NebApp $v$ & (1) Go G & \\
\hline Getting Started 5 & Q Latest Headlines & & & \\
\hline Wine Li & ist & & & \\
\hline Wine name & Wine maker & Vineyard & Year Rating $\mid$ & Info \\
\hline Semillion & All Saints & Late harvest & 19974 & (i) \\
\hline Shiraz & Baily and Baily & The Regional Varietal Range & 20044 & (i) \\
\hline Cab Sav Merlot & Beringer Blass & Shareholders Reserve & 19995 & (i) \\
\hline Cab Sav & Brand & Coonawarra & 19993 & (i) \\
\hline Cab Sav & Bremerton & Rebecca Willson & 20010 & (i) \\
\hline Shiraz & Coriole & McLaren Vale & 20003 & (i) \\
\hline Shiraz & Goodchild & Eden Valley & 19973 & (i) \\
\hline Merlot & Gossips & Warburn Estate & 20054 & (i) \\
\hline Zinfandel & Kangarilla Road & McLaren Vale & 20004 & (i) \\
\hline Dry Red & Ryecroft & Belltress Classic & 19972 & (i) \\
\hline
\end{tabular}

Fig 9. List of wines retrieved from database

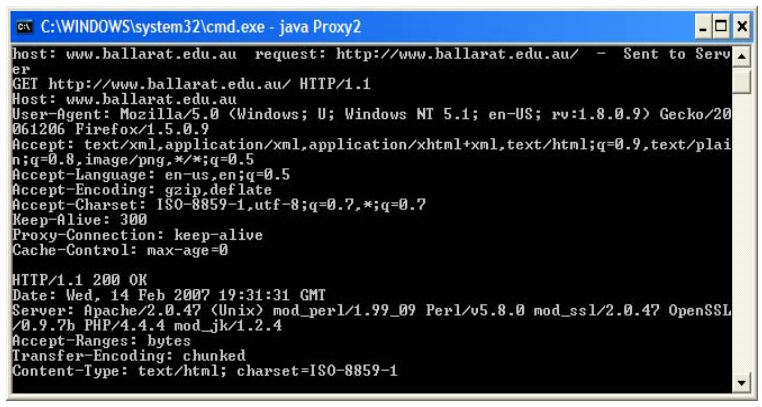

Fig 10. HTTP Request and Response

\section{Related Work}

Past research shows that proxy can be used as services [8].

\subsection{Web Services Transactions}

Web Services transaction has following properties [4]:
a. Transaction across different vendors
b. Long running transactions
c. Atomicity
d. Interactive transactions
e. Open ended transactions

Obtaining consensus and general architecture are common features of Web Services Transactions. They share the common notion of a transaction coordinator, participants, and a transaction context [10].

\subsection{Transaction Model}

Transactional model guarantees the ACID properties. Since traditional model being inadequate there were nontraditional applications like CAD/CAM and CASE etc. To model the business processes, workflow model was proposed. Some of the transaction models proposed latter was:
a. Nested Transactions
b. Open Nested Transactions
c. Saga Transaction model
d. Split-Join Transaction model
e. Long-running activity
f. Kangaroo Transaction model
g. Moflex Transaction model
h. PRO MOTION Transaction model
i. Toggle Transaction

Most of the work were done in the academic research project where as very few implemented in commercial and industry. Some of the above transaction models were proposed for mobile contexts such as Long-running activity, Kangaroo Transaction model, Toggle Transaction. [2] and [3]. 


\subsection{Transaction Protocol}

\subsubsection{The Business Transaction Protocol (BTP)}

The Business Transaction Protocol (BTP) is developed by the Business Transactions Technical Committee (BTTC) of the Organization for the Advancement of Structured Information Standards (OASIS). It is a protocol based on two phase commit which works under loosely couple environment such as Web Services. It is a XML based protocol which defines roles of transaction participants over the Internet. It was aimed at business-to-business transactions in loosely coupled domain. The protocol is very useful in Web Services environment. BTP can be layered over any transport technology, such as the Simple Object Access Protocol (SOAP), ebXML messaging [5].

\subsubsection{Transaction Internet Protocol (TIP)}

It is a transport protocol enabling distributed transaction coordinators. It is a standard protocol of the IETF. It has the ability to synchronise distributed Internet application using transactions. It is not bound with one specific transaction model. It is used in Microsoft Transaction Server (MTS) [9].

\subsubsection{Tentative Hold Protocol (THP)}

It is a message-based framework for information exchange between entities. It is an open and loosely coupled messaging framework. It provides tentative and non-blocking holds services, which is important for managing business resources in transactions. The main objective of this protocol is to increase effectiveness in automating inter-business transactions in heterogeneous environment [9].

\subsubsection{WS-Coordination and WS-Transaction}

Web Services Coordination (WS-C) and Web Services Transaction (WS-Tx) specifications were released by IBM, Microsoft and BEA in 2002. Later these specifications were updated and split into WS-Atomic Transaction and WS-BusinessActivity. WS-C defines coordination infrastructure in Web Services environment. So protocol helps to coordinate client, server and participants. WS-T defines managing and coordination of transactions [11].

\subsection{Transaction Processing Systems}

Transaction processing is a critical technology which applies across wide ranges of domain such as medical, financial and e-commerce systems etc. Each domain has its own requirements. In this section, we will survey popular transaction processing systems. Following transaction processing systems are covered:

Vendor's name is included inside the parenthesis.

CICS (IBM)

IMS (IBM)

Tuxedo (BEA)
ACMS (Digital Equipment Corp)

Encina (Transarc)

TOP END (AT\&T/NCR)

Pathway/TS (Tandem)

Microsoft Transaction Server (Microsoft)

All the given transaction processing systems have common in main features in many ways. Some of the similarities are such as multithreaded transaction servers, independent transaction servers, two-phase commit protocol, and queued TP. Differences are level of capabilities, platform coverage, programming interfaces and communication paradigm. All are three-tier architectural design with some variety [1].

Table 1. Summary of different Transaction Processing Systems

\begin{tabular}{|c|c|}
\hline$T P S$ & Main features \\
\hline CICS (IBM) & $\begin{array}{l}\text { CICS (Customer Information } \\
\text { Control System) is a transaction } \\
\text { server from IBM which runs on } \\
\text { mainframe systems under z/OS or } \\
\text { z/VSE. CICS on distributed } \\
\text { platform is known as TXSeries } \\
\text { which comes under different } \\
\text { operating systems. It is used in } \\
\text { banking and flight reservation } \\
\text { systems. }\end{array}$ \\
\hline IMS (IBM) & $\begin{array}{l}\text { Information Management System } \\
\text { (IMS) is a transaction \& database } \\
\text { management system from IBM. } \\
\text { IMS DB is organized } \\
\text { hierarchically. }\end{array}$ \\
\hline Tuxedo (BEA) & $\begin{array}{l}\text { It is high performance distributed } \\
\text { transaction management system. }\end{array}$ \\
\hline $\begin{array}{l}\text { ACMS (Digital Equipment } \\
\text { Corp) }\end{array}$ & $\begin{array}{l}\text { It uses application integration } \\
\text { framework using VAX } \\
\text { architecture. It is based on three- } \\
\text { process model - presentation, } \\
\text { workflow and data access. }\end{array}$ \\
\hline Encina (Transarc) & $\begin{array}{l}\text { Its architecture is based on the } \\
\text { OSF DCE. It uses transactional } \\
\text { RPC mechanism with nested } \\
\text { transaction. }\end{array}$ \\
\hline TOP END (AT\&T/NCR) & $\begin{array}{l}\text { It provides a flat system } \\
\text { management structure. System } \\
\text { servers communicate via node } \\
\text { manager. It has application } \\
\text { distribution functionality. }\end{array}$ \\
\hline Pathway/TS (Tandem) & $\begin{array}{l}\text { It runs on fault-tolerant Tandem } \\
\text { platform. Terminal control } \\
\text { programs are multithreaded. It } \\
\text { has loosely coupled } \\
\text { multiprocessor nodes. }\end{array}$ \\
\hline $\begin{array}{l}\text { Microsoft } \quad \text { Transaction } \\
\text { Server (Microsoft) }\end{array}$ & $\begin{array}{l}\text { It is integrated with Microsoft's } \\
\text { Component Object Model. } \\
\text { Variety of languages and OLE } \\
\text { can be used for application } \\
\text { development. It includes separate } \\
\text { transaction manager, Microsoft } \\
\text { Distributed Transaction } \\
\text { Coordinator. }\end{array}$ \\
\hline
\end{tabular}




\section{Conclusions and Future Work}

Web services are major technological trend for tomorrow's heterogeneous networked computing. There are still many issues to be resolved such as interoperability, security etc. New Web Services framework and middleware likes sProxy help to address above issues. In this working paper, we proposed a model using sProxy as the middleware. Proxies are useful to invoke Web Services to allow an easy programming model that facilitates the serialization and transmission of service and transactions invocations. We are in the preliminary state of this prototype and currently working to make full featured sProxy with transactional properties.

\section{References}

[1] P. A. Bernstein, and E. Newcomer, Principles of Transaction Processing. San Francisco: Morgan Kaufmann, 1997.

[2] R. R. Hirsch, "A Framework for Analyzing Mobile Transaction Models." Journal of database management, vol. 12, (3), p. 36, 2001.

[3] T. Jin, and S. Goschnikc, "Utilizing Web Services in an Agent Based Transaction Model (ABT)" in the 1st International Workshop on Web Services and Agent-based Engineering (WSABE'2003), 2003.

[4] B. Limthanmaphon, and Y. Zhang. "Web Service Composition Transaction Management" in Fifteenth Australasian Database Conference (ADC2004), 2004, pp. 171-179.

[5] M. Little, "Transactions and Web Services." Communications of the ACM, vol. 46, (10), pp. 49-54, 2003.

[6] M. C. Little, et al. "Constructing Reliable Web Applications using Atomic Actions," in Proceedings of the 6th International World Wide Web Conference, 1997, pp. 1281-1290.

[7] D. Malandrino, and V. Scarano. "A Taxonomy of Programmable HTTP Proxies for Advanced Edge," in Proceedings of the First International Conference on Web Information Systems and Technologies, INSTICC Press, 2005, pp. 231-238.

[8] C. H. Rao, et al., "iMobile: A Proxy-Based platform for Mobile Services," in Proceedings of the first workshop on Wireless mobile internet, Rome, Italy, New York: ACM Press, 2001, pp.3-10.

[9] B. A. Schmit, and S. Dustdar, "Towards Transactional Web Services," in Seventh IEEE International Conference on E-commerce Technology Workshops, 2005, pp. 12-20.

[10] R. S. Wu, and S.-M. Yuan, "Enable Transaction in Web Services Environment," in International Conference on Next Generation Web Services Practices (NWeSP'05), 2005, pp. 15-20.

[11] Little, M., J. Maron, and G. Pavlik, Java Transaction Processing Design and Implementation, Upper Saddle River, New Jersey: Prentice Hall, 2004.

[12] A. Luotonen, Web Proxy Servers, Upper Saddle River, NJ: Prentice Hall, 1998.

[13] K.P. Birman, Reliable Distributed Systems, New York: Springer, 2005 